\title{
A comparative study of time domain BEM for 3D elastodynamic analysis
}

\author{
Yuan Li \& Jianming Zhang \\ State Key Laboratory of Advanced Design and Manufacturing \\ for Vehicle Body, Hunan University, China
}

\begin{abstract}
In recent decades, intensive research efforts have been oriented towards the boundary element method (BEM) for 3D elastodynamic analysis, and a wealth of literature has been published. Many methods of improving the numerical stability of the time domain BEM have been proposed. These methods can be categorized into three groups: the linear $\theta$ method, the new fundamental solution method, and the convolution quadrature method. In this paper, we carried out a comparative study of these methods theoretically and numerically. Three typical methods, each of which represents the methods of the three groups respectively, have been selected and implemented. And an example problem of wave propagation in a 3D slender beam has been solved by the three methods separately. Results demonstrated that the convolution quadrature method outperforms the others with respect to both stability and accuracy.

Keywords: time domain BEM, transient elastodynamic analysis, numerical stability.
\end{abstract}

\section{Introduction}

Time domain BEM has become a widely used numerical tool to analyze elastodynamic problems over the past 30 years. As early as 1986, Banerjee et al. [1] and Manolis and Beskos [2] presented the direct BEM formulation for transient analysis of three dimensional solids, which is the most widely used and classical formulation at present. Based on the space and time dependent fundamental solution, this formulation employs higher order shape functions to approximate the field quantities in space as well as in time, and a combination of 
analytical time integration and numerical spatial integration is carried out to form a system of linear equations.

Numerical results have shown that the standard BEM formulation may be unstable in some applications. This phenomenon was first mentioned in a paper of Cole et al. [3]. Then an in depth study on this unstable behavior was carried out by Dominguez and Gallego [4]. Conclusions are summarized as: a too small time-step may cause instability in the numerical scheme, whilst a too large timestep may give rise to strong numerical damping of the results. Birgisson et al. [5] provide some evidence and define these phenomena as 'intermittent'. In his paper, it is pointed that the use of numerical integration schemes may reduce the stability. For example, if an inadequate number of sub-elements or Gauss points is used, then errors due to small violations in causality will accumulate resulting in numerical instabilities. In the opinion of Frangi [6], one of the main causes of instabilities, is identified in the interpolation adopted for displacements and tractions, which is not capable of properly simulating wave front propagation and, thus, of respecting the causality condition for the real fields. Subsequently, Frangi created "causal" shape functions in the time domain BEM for 2D elastodynamic problems.

In recent years, several approaches have been proposed to improve the stability. Among them, three ways should be mentioned as follows:

1) The first one is called the 'linear $\theta$ method' which employs modified numerical time marching procedures proposed by Araujo et al. [7]. They achieved more stable results by introducing weighting integrals. Other similar and effective schemes include: the $\varepsilon$ method [5], the half-step method [5], the constant velocity prediction method [8], and the $\alpha-\delta$ method [9].

All of these methods obtain more stable results by increasing the size of the current time step. In a paper by Walker et al. [10], it is shown that it is the eigenvalues of matrix $A$ (in the equation $X^{k+1}=A X^{k}$ ) which determine the longterm stability behavior. When the eigenvalue of matrix $\mathrm{A}$ is above one, the $X^{k}$ will, in general, rise without limit as $k$ increases. The maximum eigenvalue was found to decline with the increase of the time step length. Therefore, a better stability behavior will be observed when we use a larger time step length. This phenomenon will be shown through a numerical example in this paper later.

2) The second one is called 'new fundamental solution method' which employs a modified time dependent fundamental solution proposed by Coda and Venturini [11]. The traditional fundamental solution is a particular case of the Stokes' state, assuming that the load is a unit concentrated impulse applied at a single instant. In the work of Coda, another assumption regarding the load choice is made: a unit impulse distributed along an interval of time was taken to derive the necessary fundamental values. The details can be found in section 3 .

Another similar scheme which should be mentioned is using a new boundary integral equation (BIE) based on the velocity reciprocal theorem to reduce instability in BEM formulations, proposed by Panagiotopoulos and Manolis [12]. This BIE is established in terms of velocities and tractions instead of the traditional one which is in terms of displacements and tractions. 
3) The third one is called the 'convolution quadrature method' based on the theorem proposed by Lubich $[13,14]$ in 1988 . Then it was developed to time domain BEM by Schanz and Antes [15] in 1997. In this formulation, the convolution integral is numerically approximated by a quadrature formula whose weights are determined by the Laplace transformed fundamental solutions and a linear multistep method. It is worth noting that the frequency domain fundamental solution can be used without the need for an inverse transformation.

In the present paper, the detailed formulations of these approaches, 'the classical method', 'the linear $\theta$ method', 'the new fundamental solution method' and 'the convolution quadrature method', are presented in section 3. After that, a numerical example of a 3D slender beam is presented to compare the performance of these methods.

\section{Boundary integral formulations}

The governing equations presented here correspond to the small displacement theory for homogeneous, isotropic, linearly elastic materials. The displacement equations of motion can be written $[1,2]$

$$
\left(c_{1}^{2}-c_{2}^{2}\right) u_{i, j i}+c_{2}^{2} u_{j, i i}+f_{j} / \rho=\ddot{u}_{j} \quad(i, j=1,2,3),
$$

where $u_{i}(x, t)$ is the displacement vector at $\mathrm{x}$ and at the time $\mathrm{t}, f_{j}$ is the body force, $\rho$ is the media density. The pressure and shear wave propagation velocities are given as

$$
c_{1}=\sqrt{\frac{\lambda+2 G}{\rho}}, c_{2}=\sqrt{\frac{G}{\rho}},
$$

in which $\lambda$ and $G$ are the Lame constants given by

$$
\lambda=\frac{v E}{(1+v)(1-2 v)}, G=\frac{E}{2(1+v)},
$$

in which $E$ and $v$ represent Young's modulus and Poisson's ratio respectively.

The fundamental solutions, employed in classical time domain BEM, are the particular case of Stokes' state in which $b_{i j}^{*}=\delta_{i j} \Delta(\tau, t) \Delta(p, q)$ is assumed. The $u_{i j}^{*}$ and $P_{i j}^{*}$ expressions which can be found in several well known works $[1,2]$ are as follows:

$$
\begin{aligned}
u_{i j}^{*}(p, \tau ; q, t)= & \frac{1}{4 \pi \rho}\left\{A \cdot t^{\prime}\left[H\left(t^{\prime}, \frac{r}{c_{1}}\right)-H\left(t^{\prime}, \frac{r}{c_{2}}\right)\right]+B \cdot \Delta\left(t^{\prime}, \frac{r}{c_{1}}\right)+C \cdot \Delta\left(t^{\prime}, \frac{r}{c_{2}}\right)\right\}, \\
P_{i j}^{*}(p, \tau ; q, t)= & \frac{1}{4 \pi}\left\{D \cdot t^{\prime}\left[H\left(t^{\prime}, \frac{r}{c_{1}}\right)-H\left(t^{\prime}, \frac{r}{c_{2}}\right)\right]+E \cdot \Delta\left(t^{\prime}, \frac{r}{c_{1}}\right)+F \cdot \Delta\left(t^{\prime}, \frac{r}{c_{2}}\right)\right. \\
& \left.+G \cdot \dot{\Delta}\left(t^{\prime}, \frac{r}{c_{1}}\right)+H \cdot \dot{\Delta}\left(t^{\prime}, \frac{r}{c_{2}}\right)\right\}
\end{aligned}
$$

where 


$$
A=\frac{1}{r^{3}}\left(3 r_{, i} r_{, j}-\delta_{i j}\right), B=\frac{r_{, i} r_{, j}}{r c_{1}^{2}}, C=\frac{1}{r c_{2}^{2}}\left(\delta_{i j}-r_{, i} r_{, j}\right),
$$

and

$$
\begin{aligned}
& D=\frac{6 c_{2}^{2}}{r^{4}}\left[-5 r_{, i} r_{, j} \frac{\partial r}{\partial n}+\delta_{i j} \frac{\partial r}{\partial n}+r_{, j} n_{i}+r_{, i} n_{j}\right] \\
& E=\frac{1}{r^{2}}\left[-r_{, i} n_{j}-2 \frac{c_{2}^{2}}{c_{1}^{2}}\left(6 r_{, i} r_{, j} \frac{\partial r}{\partial n}-\delta_{i j} \frac{\partial r}{\partial n}-r_{, j} n_{i}-2 r_{, i} n_{j}\right)\right] \\
& F=\frac{1}{r^{2}}\left[12 r_{, i} r_{, j} \frac{\partial r}{\partial n}-3 \delta_{i j} \frac{\partial r}{\partial n}-3 r_{, j} n_{i}-2 r_{, i} n_{j}\right] \\
& G=\frac{1}{r c_{1}}\left[-2 \frac{c_{2}^{2}}{c_{1}^{2}} r_{, i} r_{, j} \frac{\partial r}{\partial n}+r_{, i} n_{j}\left(2 \frac{c_{2}^{2}}{c_{1}^{2}}-1\right)\right] \\
& H=\frac{1}{r c_{2}}\left[2 r_{, i} r_{, j} \frac{\partial r}{\partial n}-r_{, j} n_{i}-\delta_{i j} \frac{\partial r}{\partial n}\right]
\end{aligned}
$$

$u_{i j}^{*}(p, \tau ; q, t)$ and $P_{i j}^{*}(p, \tau ; q, t)$ represent the displacement and traction in direction $j$ at field point $q$ and at time $t$ due to a unit concentrated load at source point $p$ in direction $i$ and at time $\tau$, respectively. In the above expressions, $t^{\prime}=t-\tau$, $r_{i}=q_{i}-p_{i}, H$ is the Heaviside function and $\Delta$ is the Dirac delta function.

For zero initial conditions and zero body forces, the boundary integral formulation for transient elastodynamics can be expressed as

$$
C_{i j}(p) u_{j}(p, t)=\int_{s} u_{i j}^{*}(p, q ; t)^{*} p_{j}(q, t) d S(q)-\int_{s} p_{i j}^{*}(p, q ; t) * u_{j}(q, t) d S(q),
$$

where

$$
\begin{gathered}
u_{i j}^{*}(p, q ; t) * p_{j}(q, t)=\int_{0}^{t} u_{i j}^{s}(p, \tau ; q, t) p_{j}(q, \tau) d \tau, \\
p_{i j}^{*}(p, q ; t) * u_{j}(q, t)=\int_{0}^{t} p_{i j}^{s}(p, \tau ; q, t) u_{j}(q, \tau) d \tau,
\end{gathered}
$$

and $C_{i j}=\frac{\delta_{i j}}{2}$ on smooth surfaces.

\section{Numerical implementation}

\subsection{Time discretization}

1) Classical method

The time interval of interest $[0, t]$ is discretized into $\mathrm{N}$ time steps of duration $\Delta t$, so that $t_{M}=M \Delta t$, where $M=1,2, \ldots, N$. Boundary displacements and tractions can be linearly interpolated within a certain time step $M$ as given below:

$$
\begin{aligned}
& u_{i}(q, \tau)=\sum_{m=1}^{M}\left[N^{m-1} u_{i}^{m-1}(q)+N^{m} u_{i}^{m}(q)\right], \\
& p_{i}(q, \tau)=\sum_{m=1}^{M}\left[N^{m-1} p_{i}^{m-1}(q)+N^{m} p_{i}^{m}(q)\right],
\end{aligned}
$$


where

$$
\begin{gathered}
N^{m-1}=\frac{m \Delta t-\tau}{\Delta t} \Phi_{m}(\tau), N^{m}=\frac{\tau-(m-1) \Delta t}{\Delta t} \Phi_{m}(\tau), \\
\Phi_{m}(\tau)=H(\tau-(m-1) \Delta t)-H(\tau-m \Delta t) .
\end{gathered}
$$

Then the following time discretized boundary integral equation is obtained:

$$
C_{i j}(p) u_{j}^{M}(p)=\sum_{m=1}^{M} \int_{S}\left(U_{i j}^{m}(p, q)-P_{i j}^{m}(p, q)\right) d S(q),
$$

where

$$
\begin{aligned}
& U_{i j}^{m}(p, q)=\int_{(m-1) \Delta t}^{m \Delta t} u_{i j}^{*}(p, q ; M \Delta t-\tau)\left(\frac{m \Delta t-\tau}{\Delta t} p_{j}^{m-1}(q)+\frac{\tau-(m-1) \Delta t}{\Delta t} p_{j}^{m}(q)\right) d \tau \\
& P_{i j}^{m}(p, q)=\int_{(m-1) \Delta t}^{m \Delta t} p_{i j}^{*}(p, q ; M \Delta t-\tau)\left(\frac{m \Delta t-\tau}{\Delta t} u_{j}^{m-1}(q)+\frac{\tau-(m-1) \Delta t}{\Delta t} u_{j}^{m}(q)\right) d \tau
\end{aligned} .
$$

The above process is the traditional time domain method used to deal with the time convolution. Here we will introduce three different measures taken to reduce the instability.

2) Linear $\theta$ method

In the linear $\theta$ method, the latter time response is first evaluated at time $t=(M-1+\theta) \Delta t \quad(\theta \geq 1.0)$, and subsequently the solution at time $t=M \Delta t$ is obtained by linear interpolation as follows [7]:

$$
u_{i}^{M}=\frac{1}{\theta} u_{i}^{M+\theta-1}+\frac{\theta-1}{\theta} u_{i}^{M-1}, \quad p_{i}^{M}=\frac{1}{\theta} p_{i}^{M+\theta-1}+\frac{\theta-1}{\theta} p_{i}^{M-1} .
$$

Then, Eqn. (11) can be re-expressed as:

$$
\begin{aligned}
C_{i j}(p) u_{j}^{M+\theta-1}(p) & =\int_{S}\left(U_{i j}^{M+\theta-1}(p, q)-P_{i j}^{M+\theta-1}(p, q)\right) d S(q) \\
& +\sum_{m=1}^{M-1} \int_{S}\left(U_{i j}^{m}(p, q)-P_{i j}^{m}(p, q)\right) d S(q),
\end{aligned}
$$

where

$$
\begin{gathered}
U_{i j}^{M+\theta-1}=\int_{(M-1) \Delta t}^{(M+\theta-1) \Delta t} u_{i j}^{*}(p, q ;(M+\theta-1) \Delta t-\tau)\left(\frac{(M+\theta-1) \Delta t-\tau}{\theta \Delta t} p_{j}^{M-1}(q)\right. \\
\left.+\frac{\tau-(M-1) \Delta t}{\theta \Delta t} p_{j}^{M+\theta-1}(q)\right) d \tau \\
P_{i j}^{M+\theta-1}=\int_{(M-1) \Delta t}^{(M+\theta-1) \Delta t} p_{i j}^{*}(p, q ;(M+\theta-1) \Delta t-\tau)\left(\frac{(M+\theta-1) \Delta t-\tau}{\theta \Delta t} u_{j}^{M-1}(q)\right. \\
\left.+\frac{\tau-(M-1) \Delta t}{\theta \Delta t} u_{j}^{M+\theta-1}(q)\right) d \tau \\
U_{i j}^{m}=\int_{(m-1) \Delta t}^{m \Delta t} u_{i j}^{*}(p, q ;(M+\theta-1) \Delta t-\tau)\left(\frac{m \Delta t-\tau}{\Delta t} p_{j}^{m-1}(q)+\frac{\tau-(m-1) \Delta t}{\Delta t} p_{j}^{m}(q)\right) d \tau \\
P_{i j}^{m}=\int_{(m-1) \Delta t}^{m \Delta t} p_{i j}^{*}(p, q ;(M+\theta-1) \Delta t-\tau)\left(\frac{m \Delta t-\tau}{\Delta t} u_{j}^{m-1}(q)+\frac{\tau-(m-1) \Delta t}{\Delta t} u_{j}^{m}(q)\right) d \tau
\end{gathered}
$$

Time integration in Eqn. (14) can be done analytically. It should be noted that the time step length is equal to $\theta \Delta t$ in the M-step, which in the previous steps is 
equal to $\Delta t$. When $\theta=1.0$, the linear $\theta$ procedure is same with the traditional one.

3) New fundamental solution method

Trying to obtain an alternative which could improve the stability behavior for BEM transient analysis, Coda and Venturini [11] use another load function given by the following expression:

$$
b_{i j}^{*}=\delta_{i j} \Delta(p, q)[H(\tau, t)-H(\tau, t-\Delta t)] / \Delta t,
$$

The $u_{i j}^{*}$ and $P_{i j}^{*}$ expressions become:

$$
\begin{aligned}
& u_{i j}^{*}(p, \tau ; q, t)=\frac{1}{4 \pi \rho \Delta t}\left[f_{i j}^{*}(p, \tau ; q, t)-f_{i j}^{*}(p, \tau ; q, t-\Delta t)\right], \\
& p_{i j}^{*}(p, \tau ; q, t)=\frac{1}{4 \pi \Delta t}\left[g_{i j}^{*}(p, \tau ; q, t)-g_{i j}^{*}(p, \tau ; q, t-\Delta t)\right],
\end{aligned}
$$

where

$$
\begin{gathered}
f_{i j}^{*}=0.5 A \cdot\left[\left(t^{2}-\frac{r^{2}}{c_{1}^{2}}\right) H\left(t^{\prime}, \frac{r}{c_{1}}\right)-\left(t^{\prime 2}-\frac{r^{2}}{c_{2}^{2}}\right) H\left(t^{\prime}, \frac{r}{c_{2}}\right)\right]+B \cdot H\left(t, \frac{r}{c_{1}}\right)+C \cdot H\left(t^{\prime}, \frac{r}{c_{2}}\right), \\
g_{i j}^{*}=0.5 D \cdot\left[\left(t^{\prime 2}-\frac{r^{2}}{c_{1}^{2}}\right) H\left(t^{\prime}, \frac{r}{c_{1}}\right)-\left(t^{2}-\frac{r^{2}}{c_{2}^{2}}\right) H\left(t^{\prime}, \frac{r}{c_{2}}\right)\right]+E \cdot H\left(t^{\prime}, \frac{r}{c_{1}}\right) \\
+F \cdot H\left(t^{\prime}, \frac{r}{c_{2}}\right)+G \cdot \Delta\left(t, \frac{r}{c_{1}}\right)+H \cdot \Delta\left(t, \frac{r}{c_{2}}\right)
\end{gathered}
$$

All of the parameters and polynomials in expression (19) are the same with that defined in previous fundamental solutions (4) and (5). For zero initial conditions and zero body forces, the new boundary integral formulation for transient elastodynamics can be expressed as:

$$
C_{i j}(p) \int_{t-\Delta t}^{t} \frac{u_{j}(p, \tau)}{\Delta t} d \tau=\int_{s} u_{i j}^{*}(p, q ; t) * p_{j}(q, t) d S(q)-\int_{s} p_{i j}^{*}(p, q ; t) * u_{j}(q, t) d S(q) .
$$

As usual, the time interval of interest $[0, t]$ is discretized into $N$ time steps of duration $\Delta t$. This new fundamental solutions permit the use of the constant time approximation for displacements, because it does not have terms containing the Dirac's delta derivatives. For the particular case of the time discretization, constant elements have been adopted in this paper. Thus, Eqn. (20) can be written as follows:

$$
\begin{aligned}
C_{i j}(p) u_{j}^{M}(p) & =\sum_{m=1}^{M} \int_{S} \int_{(m-1) \Delta t}^{m \Delta t} u_{i j}^{s}(p, q ; M \Delta t-\tau) p_{j}^{m}(q) d \tau d S(q) \\
& -\sum_{m=1}^{M} \int_{S} \int_{(m-1) \Delta t}^{m \Delta t} p_{i j}^{s}(p, q ; M \Delta t-\tau) u_{j}^{m}(q) d \tau d S(q)
\end{aligned}
$$

where

$$
M=1,2, \ldots, N
$$

Then time integration in Eqn. (21) can be done analytically like previous boundary integration equations. 
4) Convolution quadrature method

Unlike dealing with the time integration analytically in time interval $[t, t+\Delta t]$ in the first three methods, the convolution between the fundamental solutions and the corresponding nodal values in Eqn. (7) is performed numerically with the socalled 'convolution quadrature method' in this formulation. A convolution integral of the form

$$
y(t)=f(t) * g(t)=\int_{0}^{t} f(t-\tau) g(\tau) d \tau
$$

can be approximated by the finite sum

$$
y(M \Delta t)=\sum_{m=0}^{M} \omega_{M-m}(\Delta t) g(m \Delta t), \quad(M=0,1, \ldots, N) .
$$

The integration weights $\omega_{M}(\Delta t)$ are the coefficients of the power series for the function $\hat{f}(\Gamma(z) / \Delta t)$ at the point $\gamma(z) / \Delta t$. The coefficients $\omega_{M}(\Delta t)$ are calculated by the integral

$$
\omega_{M}(\Delta t)=\frac{1}{2 \pi i} \int_{|z|=\Re} \hat{f}\left(\frac{\gamma(z)}{\Delta t}\right) z^{-M-1} d z \approx \frac{\Re^{-M}}{L} \sum_{l=0}^{L-1} \hat{f}\left(\frac{\gamma\left(\Re e^{i l \frac{2 \pi}{L}}\right)}{\Delta t}\right) e^{-i l M \frac{2 \pi}{L}} .
$$

Here, $\gamma(z)$ is the quotient of the characteristic polynomials of a linear multistep method, for example, the backward differentiation formula of second order $\gamma(z)=1.5-2 z+0.5 z^{2}$. If one assumes that the values of $\hat{f}(z)$ in Eqn. (25) are computed with an error bounded by $\varepsilon\left(\varepsilon>=10^{-10}\right)$, then the choice $L=N+1$ and $\mathfrak{R}^{L}=\sqrt{\varepsilon}$ yields an error in $\omega_{M}(\Delta t)$ of size $\mathrm{O}(\sqrt{\varepsilon})$. Details of the derivation process can be found in reference [15].

The quadrature formula (24) is applied to Eqn. (7). The result is the following boundary element time-stepping formulation for $M=0,1,2, \ldots, N$

$$
C_{i j}(p) u_{j}^{M}(p)=\sum_{m=0}^{M} \int_{S}\left\{\omega_{M-m}\left(\hat{u}_{i j}^{*}, p, \Delta t\right) p_{j}^{m}(q)-\omega_{M-m}\left(\hat{p}_{i j}^{*}, p, \Delta t\right) u_{j}^{m}(q)\right\} d S(q)
$$

with the weights corresponding to Eqn. (25)

$$
\begin{aligned}
& \omega_{M}\left(\hat{u}_{i j}^{*}, p, \Delta t\right)=\frac{\mathfrak{R}^{-M}}{L} \sum_{l=0}^{L-1} \hat{u}_{i j}^{*}\left(p, q, \frac{\gamma\left(\Re e^{i l \frac{2 \pi}{L}}\right)}{\Delta t}\right) e^{-i l M \frac{2 \pi}{L}}, \\
& \omega_{M}\left(\hat{p}_{i j}^{*}, p, \Delta t\right)=\frac{\mathfrak{R}^{-M}}{L} \sum_{l=0}^{L-1} \hat{p}_{i j}^{*}\left(p, q, \frac{\gamma\left(\Re e^{i l \frac{2 \pi}{L}}\right)}{\Delta t}\right) e^{-i l M \frac{2 \pi}{L}} .
\end{aligned}
$$

Note that the calculation of the quadrature weights (27) and (28) is based on the Laplace transformed fundamental solutions as follows:

$$
\begin{aligned}
\hat{u}_{i j}^{*}(p, q, s) & =\frac{1}{4 \pi \rho}\left\{A \cdot\left[\frac{s r / c_{1}+1}{s^{2}} e^{-s r / c_{1}}-\frac{s r / c_{2}+1}{s^{2}} e^{-s r / c_{2}}\right]+B \cdot e^{-s r / c_{1}}+C \cdot e^{-s r / c_{2}}\right\}, \\
\hat{p}_{i j}^{*}(p, q, s) & =\frac{1}{4 \pi}\left\{D \cdot\left[\frac{s r / c_{1}+1}{s^{2}} e^{-s r / c_{1}}-\frac{s r / c_{2}+1}{s^{2}} e^{-s r / c_{2}}\right]+E \cdot e^{-s r / c_{1}}+F \cdot e^{-s r / c_{2}}\right. \\
& \left.+G \cdot s e^{-s r / c_{1}}+H \cdot s e^{-s r / c_{2}}\right\}
\end{aligned}
$$

The polynomial expressions of $\mathrm{A} \sim \mathrm{H}$ are the same with expression (6). 


\subsection{Spatial discretization}

Note that we adopt the same way of spatial discretization and the interpolation functions for these four methods in this paper. Assume that the boundary is divided into $E$ elements. The value of displacements $u_{j}^{m}$ and tractions $p_{j}^{m}$ can be approximated using the $K$-order interpolation functions:

$$
u_{j}^{m}=\sum_{e=1}^{E} \sum_{k=1}^{K} \Phi^{e k} u_{j}^{e k m}, \quad p_{j}^{m}=\sum_{e=1}^{E} \sum_{k=1}^{K} \Phi^{e k} p_{j}^{e k m} .
$$

Then, insert (31) in Eqn. (11), (14), (21), (26) respectively. As for any boundary element formulation, the integral representation is transformed into an algebraic equation, after carrying out all space and time integrals over elements defined by both discretizations. A system of linear equations is achieved as follows:

$$
H^{M M} u^{M}=G^{M M} p^{M}+\sum_{m=1}^{M-1}\left(G^{M m} p^{m}-H^{M m} u^{m}\right) \quad(M=1,2, \ldots, N) .
$$

A strong singularity appears in the traction fundamental solution when $M=m$. In such case the $H^{M M}$ matrix is not computed by direct integration, it is obtained using the static rigid body method [2].

\section{Numerical examples}

It should be illustrated that all the implementations of these methods are performed in the boundary face method (BFM, proposed by Zhang et al. [16]) frame which is unified with CAD software Unigraphics NX of edition 4.0. For time dependent problems. Zhou et al. [17] and Guo et al. [18] have extended the $\mathrm{BFM}$ for a $3 \mathrm{D}$ transient heat conduction problem.

In order to compare the numerical stability and accuracy of these methods, a slender beam under a Heaviside-type force $p=1000 \mathrm{~N} / \mathrm{m}^{2}$ shown in Fig. 1(b) will be modelled in this section. Young's modulus $E=1.1 \times 10^{5} \mathrm{~N} / \mathrm{m}^{2}$, Poisson's ratio $v=0.0$ and the mass density $\rho=2.0 \mathrm{~kg} / \mathrm{m}^{3}$. The geometry data of the beam are $L=8.0 m, W=H=2.0 \mathrm{~m}$. We employ in total 117 nodes and 72 linear quadrilateral elements which are discontinuous between the adjacent surfaces. The elements and nodes distribution are illustrated in Fig. 1(a).

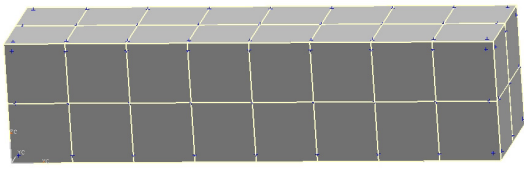

(a) Spatial discretization

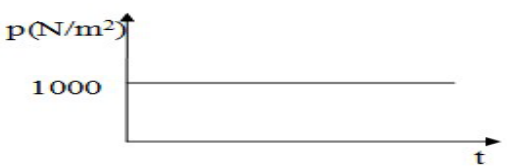

(b) Heaviside-type loading

Figure 1: Slender beam. 
Depending on the different characteristics of these methods, different time shape functions are adopted. Linear time interpolation for displacements and constant time approximation for tractions are employed for the classical method. Linear time interpolation for displacements and tractions are employed for the linear $\theta$ method, and $\theta=1.5$ in this application. Constant time approximation for displacements and tractions are employed for the new fundamental solution method. Values of the parameters in the convolution quadrature method are

$$
L=N+1, \quad \Re^{L}=\sqrt{\varepsilon}\left(\varepsilon=10^{-10}\right), \gamma(z)=1.5-2 z+0.5 z^{2}
$$

respectively.

The time step length control parameter $\beta$ used in this paper is defined as $\beta=c_{1} \Delta t / d$, where $d$ is the characteristic element length. In this example we adopt three sets of $\beta$ as follows:

$$
\begin{aligned}
& \text { (1) } d=1 m, \Delta t=0.00712 s, \beta=0.8, \\
& \text { (2) } d=1 m, \Delta t=0.00356 s, \beta=0.4, \\
& \text { (3) } d=1 m, \Delta t=0.00089 s, \beta=0.2 .
\end{aligned}
$$

The displacements at the beam free end achieved for the above methods, including the classical method (CM), the linear $\theta$ method (LTM), the new fundamental solution method (NFSM) and the convolution quadrature method (CQM), are shown in Figs 2(a)-4(a). The corresponding tractions at the fixed end also are given in Figs 2(b)-4(b).

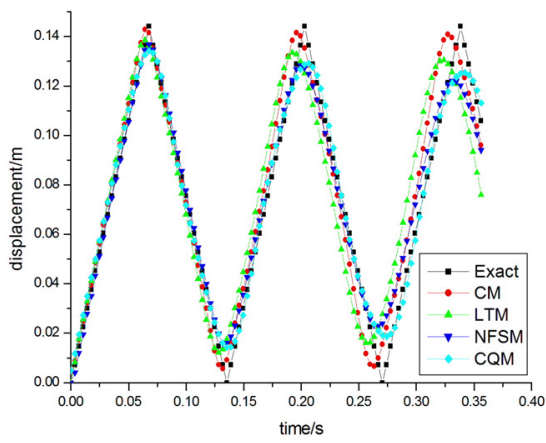

(a) Displacements at the beam free end

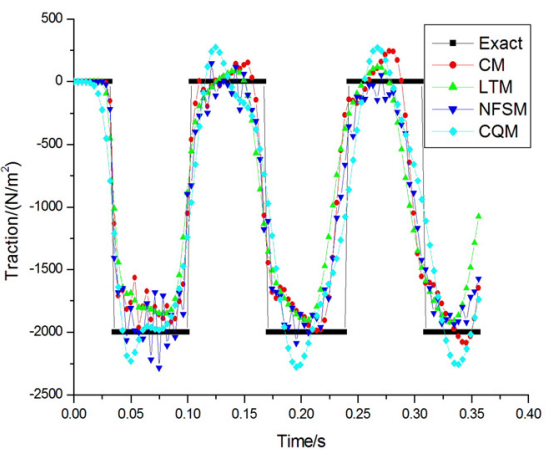

(b) Tractions at the beam fixed end

Figure 2: $\quad$ Results with $\beta=0.8$.

As shown in Figs 2-4, a proper choice of parameter $\beta$ is important for time domain BEM. By observing the displacement curve when $\beta=0.8$, we found that the results of all of these methods keep stability during the concerning time period, the decay rate of these four schemes in descending order are: CM, LTM, NSFM, CQM. It means that the accuracy of CQM is maintained more stable compared to other methods. When $\beta$ becomes 0.4 or a lower value, results of 


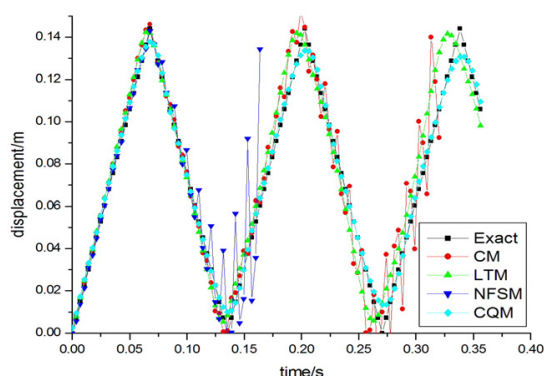

(a) Displacements at the beam free end

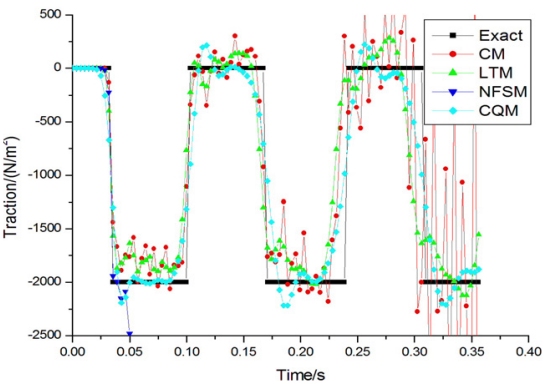

(b) Tractions at the beam fixed end

Figure 3: $\quad$ Results with $\beta=0.4$.

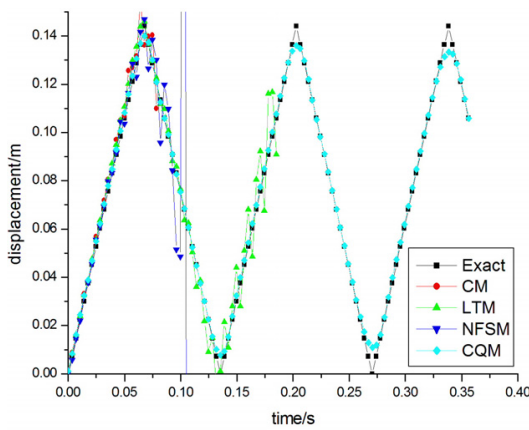

(a) Displacements at the beam free end

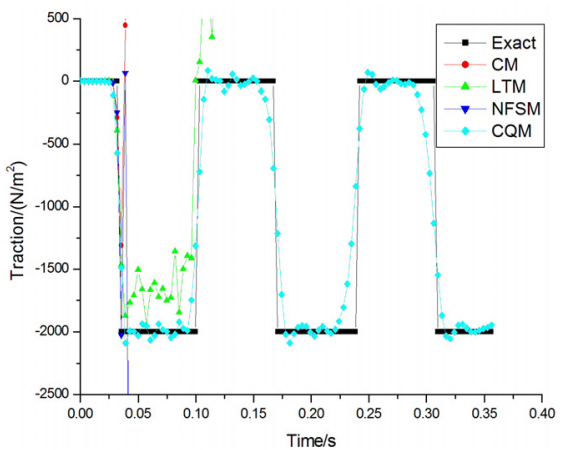

(b) Tractions at the beam fixed end

Figure 4: $\quad$ Results with $\beta=0.2$.

these methods except CQM become unstable with the increase of response time. According to the above phenomenon conclusions can be summed up as follows:

- LTM: Compared to the classical method, LTM improves the stability effectively by adopting a $\theta$ factor to increase the step length of the current time step. However, the accuracy of results reduced with the increase of $\theta$ value.

- NSFM: Compared to the classical method, NSFM reduces the decay rate of results effectively by adopting a new load function including the Heaviside function. However, the stability of results is not improved.

- CQM: Compared to the classical method and other two methods, CQM not only improves the stability of results effectively, but also reduces the decay rate of results effectively by adopting a numerical approximation for the convolution integral. 


\section{Conclusions}

The stability of the time domain BEM has been intensively studied due to its importance for the method to be applied to engineering problems. So far many methods for improving the stability have been proposed. These methods can be categorized into four groups: the classical method, the linear $\theta$ method, the new fundamental solution method and the convolution quadrature method. In this paper, we have carried out a comparative study of these methods. The formulations of all these methods are presented in detail and implemented in $\mathrm{C}++$ codes. Then the stability and accuracy of these methods are compared using numerical examples. The results show that the linear $\theta$ method has some advantages with regard to stability, while the new fundamental solution method can alleviate the damping effect. Nevertheless, none of them can remain stable in the case of a very small time step length. A proper choice of the time step length and element length is still of crucial importance for them to get reasonable results. The convolution quadrature method, however, outperforms the other methods with respect to both stability and accuracy. A fast implementation of the convolution quadrature method for solving large scale problems is planned in the near future.

\section{Acknowledgement}

This work was supported by the National Science Foundation of China under grant number 11172098 .

\section{References}

[1] Banerjee P.K., Ahmad S., Manolis G.D., Transient elastodynamic analysis of three-dimensional problems by boundary element method. Earthquake Engineering and Structural Dynamics, 14, pp. 933-949, 1986.

[2] Manolis G.D., Beskos D.E., Boundary Element Method in Elastodynamics. Spon Press, 1988.

[3] Cole D.N., Kosloff D.D., Minster J.B., A numerical boundary integral equation method for elastodynamics. Bulletin of the Seismological Society of America, 68(5), pp. 1331-1357, 1978.

[4] Dominguez J., Gallego R., The time domain boundary element method for elastodynamic problems. Mathematical and Computer Modelling, 15(3-5), pp. 119-129, 1991.

[5] Birgisson B., Siebrits E., Peirce A.P., Elastodynamic direct boundary element methods with enhanced numerical stability properties. International Journal for Numerical Methods in Engineering, 46, pp. 871888, 1999.

[6] Frangi A., "Causal" shape functions in the time domain boundary element method. Computational Mechanics, 25, pp. 533-541, 2000. 
[7] Araujo F.C., Mansur W.J., Nishikava L.K., A linear $\theta$ time-marching algorithm in 3D BEM formulation for elastodynamics. Engineering Analysis with Boundary Elements, 23, pp. 825-833, 1999.

[8] Marrero M., Dominguez J., Numerical behavior of time domain BEM for three-dimensional transient elastodynamic problem. Engineering Analysis with Boundary Elements, 27, pp. 39-48, 2003.

[9] Soares D., Mansur W.J., An efficient stabilized boundary element formulation for 2D time domain acoustics and elastodynamics. Computational Mechanics, 40, pp. 355-365, 2007.

[10] Walker S.P., Bluck M.J., Chatzis I., The stability of integral equation timedomain computations for three-dimensional scattering; similarities and differences between electrodynamic and elastodynamic computations. International Journal of Numerical Modelling, 15, pp. 459-474, 2002.

[11] Coda H.B., Venturini W.S., Further improvements on three dimensional transient BEM elastodynamic analysis. Engineering Analysis with Boundary Elements, 17, pp. 231-243, 1996.

[12] Panagiotopoulos C.G., Manolis G.D., Three-dimensional BEM for transient elastodynamics based on the velocity reciprocal theorem. Engineering Analysis with Boundary Elements, 35, pp. 507-516, 2011.

[13] Lubich C., Convolution quadrature and discretized operational calculus. I. Numerische Mathematic, 52, pp. 129-145, 1988.

[14] Lubich C., Convolution quadrature and discretized operational calculus. II. Numerische Mathematic, 52, pp. 413-425, 1988.

[15] Schanz M., Antes H., Application of 'Operational Quadrature Methods' in Time Domain Boundary Element Methods. Meccanica, 32, pp. 179-186, 1997.

[16] Zhang J., Qin X. et al., A boundary face method for potential problems in three dimensions. International Journal for Numerical Methods in Engineering, 80(3), pp. 320-337, 2009.

[17] Zhou F., Xie G., Zhang J., Zheng X., Transient heat conduction analysis of solids with small open-ended tubular cavities by boundary face method. Engineering Analysis with Boundary Elements, 37, pp. 542-550, 2013.

[18] Guo S., Zhang, J. et al., Three-dimensional transient heat conduction analysis by Laplace transformation and multiple reciprocity boundary face method. Engineering Analysis with Boundary Elements, 37, pp. 15-22, 2013. 